\title{
Penguin and Box Diagrams in Unitary Gauge
}

\author{
Xiao-Gang $\mathrm{He}{ }^{*}$ and Jusak Tandean \\ Department of Physics and Center for Theoretical Sciences, \\ National Taiwan University, Taipei 106, Taiwan \\ G. Valencidid \\ Department of Physics and Astronomy, \\ Iowa State University, Ames, Iowa 50011, USA
}

(Dated: November 1, 2018)

\begin{abstract}
We evaluate one-loop diagrams in the unitary gauge that contribute to flavor-changing neutral current (FCNC) transitions involving two and four fermions. Specifically, we deal with penguin and box diagrams arising within the standard model (SM) and in nonrenormalizable extensions thereof with anomalous couplings of the $W$ boson to quarks. We show explicitly in the SM the subtle cancelation among divergences from individual unitary-gauge contributions to some of the physical FCNC amplitudes and derive expressions consistent with those obtained using $R_{\xi}$ gauges in the literature. Some of our results can be used more generally in certain models involving fermions and gauge bosons which have interactions similar in form to those we consider.
\end{abstract}

*Electronic address: hexg@phys.ntu.edu.tw

$\dagger$ Electronic address: jtandean@yahoo.com

‡Electronic address: valencia@iastate.edu 
In the presence of new physics affecting primarily the charged weak currents involving quarks, the $W$ boson may have couplings to quarks beyond those in the standard model (SM). The effective Lagrangian for a general parametrization of the $W$ boson interacting with an up-type quark $U$ and a down-type quark $D$ can be written as

$$
\mathcal{L}_{U D W}=-\bar{U} \gamma^{\mu}\left(g_{\mathrm{L}}^{D} P_{\mathrm{L}}+g_{\mathrm{R}}^{D} P_{\mathrm{R}}\right) D W_{\mu}^{+}+\text {H.c. }
$$

where $g_{\mathrm{L}, \mathrm{R}}^{D}$ are complex coupling constants and $P_{\mathrm{L}, \mathrm{R}}=\frac{1}{2}\left(1 \mp \gamma_{5}\right)$. In the SM limit, these constants become

$$
g_{\mathrm{L}}^{D}=\frac{g}{\sqrt{2}} V_{U D}, \quad g_{\mathrm{R}}^{D}=0
$$

where $g$ is the weak coupling constant and $V_{k l}$ are elements of the Cabibbo-Kobayashi-Maskawa (CKM) matrix.

Beyond the SM, the new couplings in Eq. (1) not only affect weak decays through tree-level interactions, but also modify flavor-changing neutral current (FCNC) transitions at one-loop level. In Ref. [1], we have studied several two- and four-fermion FCNC transitions induced at one-loop level by the new couplings in the charm sector and determined constraints on them from various processes. Here we provide the detailed derivation of the loop formulas summarized therein. Some of the results we present in this paper can also be applied to other models involving fermions and gauge bosons which have interactions similar in form to those we consider.

In treating the loops, we adopt the unitary gauge, which is convenient in the absence of knowledge about the new degrees of freedom. Another advantage is that, since diagrams involving unphysical states are absent in unitary gauge, the number of diagrams to deal with is smaller than that in the $R_{\xi}$ gauges usually employed in the literature. Unlike in $R_{\xi}$ gauges, our calculations in unitary gauge produce divergences in some of the individual contributions. To treat them, we employ dimensional regularization with a fully anticommuting $\gamma_{5}$. We express the divergent part of the $D$-dimensional integration in terms of the combination

$$
\Delta=\frac{2}{4-D}-\gamma_{\mathrm{E}}+\ln \frac{4 \pi \mu^{2}}{m_{W}^{2}},
$$

where $\gamma_{\mathrm{E}}$ is the Euler constant and $\mu$ the mass scale that arises from the regularization procedure. For physical processes, the divergent parts should cancel in the total amplitudes in the SM limit and the results should agree with those obtained in $R_{\xi}$ gauges. It is therefore instructive to see how the individual contributions in unitary gauge differ from those in the general $R_{\xi}$ gauge.

At the quark level, the loop-induced physical processes of interest in Ref. [1] are $d \rightarrow d^{\prime} \gamma$, $d \rightarrow d^{\prime} g, d \bar{d}^{\prime} \rightarrow \nu \bar{\nu}, d \bar{d}^{\prime} \rightarrow \ell^{+} \ell^{-}$, and $d \bar{d}^{\prime} \rightarrow \bar{d} d^{\prime}$, where $d$ and $d^{\prime}$ are down-type quarks, with $\gamma, g$, $\nu$, and $\ell$ as usual denoting a photon, gluon, neutrino, and charged lepton, respectively. The relevant diagrams are displayed in Figs. 1 and 2, where the loops in unitary gauge contain only fermions and $W$ bosons. The $d \bar{d}^{\prime} \rightarrow \nu \bar{\nu}$ and $d \bar{d}^{\prime} \rightarrow \ell^{+} \ell^{-}$transitions also receive $Z$-penguin contributions and, for the latter, $\gamma$-penguin contributions as well.

Some of our results are new, while some have been found before using unitary or $R_{\xi}$ gauges. Specific aspects of our paper not previously available in the literature include unitary-gauge expressions for individual amplitudes corresponding to the separate diagrams in Fig. 1 contributing to 


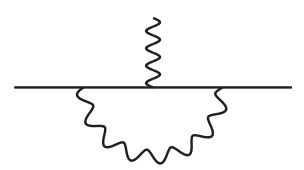

(a)

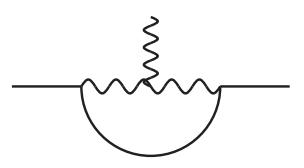

(b)
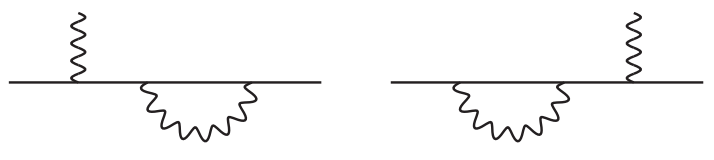

(c)

FIG. 1: Diagrams contributing to amplitudes for $d \rightarrow d^{\prime} \mathcal{V}^{*}$, with $\mathcal{V}$ being a neutral gauge boson. In all figures, straight lines denote fermions and the loops contain $W$ bosons besides fermions.

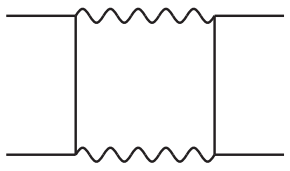

(a)

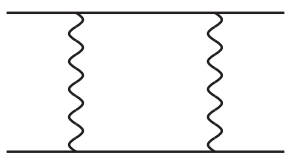

(b)

FIG. 2: Box diagrams contributing to amplitudes for (a) $d \bar{d}^{\prime} \rightarrow \nu \bar{\nu}$ or $\ell^{+} \ell^{-}$and $(\mathrm{a}, \mathrm{b}) d \bar{d}^{\prime} \rightarrow d \bar{d}^{\prime}$.

$d \rightarrow d^{\prime} \mathcal{V}^{*}$, arising from the quark- $W$ interactions of Eq. (11) and SM-like couplings of the neutral gauge bosons $(Z, \gamma, g)$ to fermions and the $W$. These particular results, presented in the Appendix, can thus be used more generally in models involving fermions and gauge bosons having interactions similar in form to those we discuss. Another new aspect of our work is the explicit demonstration, in unitary gauge, of cancelation between divergences from penguin and box diagrams contributing to $d \bar{d}^{\prime} \rightarrow \nu \bar{\nu}$ and $d \bar{d}^{\prime} \rightarrow \ell^{+} \ell^{-}$. Moreover, perhaps more completely than earlier work in the literature, we provide a set of unitary-gauge results for the FCNC transitions listed above induced by the interactions in Eq. (1), along with SM-like interactions of the neutral gauge bosons. We will compare our results with those in the literature, where available.

In the following, we derive first the amplitudes for $d \rightarrow d^{\prime} \mathcal{V}^{*}$, where $d$ and $d^{\prime}$ are on-shell, but the neutral gauge-boson $\mathcal{V}$ is off-shell with momentum $k$ that is small compared to $m_{W}$. We have collected the resulting formulas corresponding to the diagrams in Fig. 1(a), (b), and (c) in the Appendix. Then, assuming that $\mathcal{V}$ has SM interactions with fermions, the couplings being given in Eq. (A7), we combine the three contributions. Thus we obtain for $\mathcal{V}=Z$

$$
\mathcal{M}_{d \rightarrow d^{\prime} Z^{*}}^{q}=\frac{g \varepsilon_{\mu}^{*}}{16 \pi^{2} c_{\mathrm{w}}} \bar{d}^{\prime}\left[\gamma^{\mu}\left(z_{\mathrm{L}}^{q} P_{\mathrm{L}}+z_{\mathrm{R}}^{q} P_{\mathrm{R}}\right)+\frac{i \sigma^{\mu \nu} k_{\nu}}{m_{W}^{2}}\left(Z_{\mathrm{L}}^{q} P_{\mathrm{L}}+Z_{\mathrm{R}}^{q} P_{\mathrm{R}}\right)\right] d
$$

upon setting set $k^{2}=0$, where

$$
\begin{gathered}
z_{\mathrm{L}}^{q}=\frac{g_{\mathrm{L}}^{d} \bar{g}_{\mathrm{L}}^{d^{\prime}}}{4}\left[-x_{q} \Delta+\frac{7 x_{q}-x_{q}^{2}}{2\left(1-x_{q}\right)}+\frac{4 x_{q}-2 x_{q}^{2}+x_{q}^{3}}{\left(1-x_{q}\right)^{2}} \ln x_{q}\right], \\
z_{\mathrm{R}}^{q}=\frac{g_{\mathrm{R}}^{d} \bar{g}_{\mathrm{R}}^{d^{\prime}}}{4}\left[7 x_{q} \Delta+\frac{15 x_{q}-9 x_{q}^{2}}{2\left(1-x_{q}\right)}-\frac{4 x_{q}-14 x_{q}^{2}+7 x_{q}^{3}}{\left(1-x_{q}\right)^{2}} \ln x_{q}\right], \\
Z_{\mathrm{L}}^{q}=g_{\mathrm{L}}^{d} \bar{g}_{\mathrm{L}}^{d^{\prime}} m_{d^{\prime}} \mathcal{Z}_{0}^{\mathrm{SM}}\left(x_{q}\right)+g_{\mathrm{R}}^{d} \bar{g}_{\mathrm{R}}^{d^{\prime}} m_{d} z_{0}\left(x_{q}\right)+g_{\mathrm{L}}^{d} \bar{g}_{\mathrm{R}}^{d^{\prime}} m_{q} \mathcal{Z}_{0}\left(x_{q}\right), \\
Z_{\mathrm{R}}^{q}=g_{\mathrm{L}}^{d} \bar{g}_{\mathrm{L}}^{d^{\prime}} m_{d} \mathcal{Z}_{0}^{\mathrm{SM}}\left(x_{q}\right)+g_{\mathrm{R}}^{d} \bar{g}_{\mathrm{R}}^{d^{\prime}} m_{d^{\prime}} z_{0}\left(x_{q}\right)+\bar{g}_{\mathrm{L}}^{d^{\prime}} g_{\mathrm{R}}^{d} m_{q} \mathcal{Z}_{0}\left(x_{q}\right),
\end{gathered}
$$




$$
\begin{gathered}
\mathcal{Z}_{0}^{\mathrm{SM}}(x)=\frac{13 x-2 x^{2}+x^{3}}{24(1-x)^{3}}-\frac{7 x-5 x^{2}-8 x^{3}}{12(1-x)^{3}} c_{\mathrm{w}}^{2}+\frac{x+x^{2}-\left(4 x^{2}-6 x^{3}\right) c_{\mathrm{w}}^{2}}{4(1-x)^{4}} \ln x, \\
z_{0}(x)=\mathcal{Z}_{0}^{\mathrm{SM}}(x)-\frac{16 x-29 x^{2}+7 x^{3}}{24(1-x)^{3}}-\frac{2 x-3 x^{2}}{4(1-x)^{4}} \ln x \\
\mathcal{Z}_{0}(x)=\frac{5\left(4+x+x^{2}\right)-4\left(20-31 x+5 x^{2}\right) c_{\mathrm{w}}^{2}}{24(1-x)^{2}}+\frac{5 x-4\left(2 x-3 x^{2}\right) c_{\mathrm{w}}^{2}}{4(1-x)^{3}} \ln x .
\end{gathered}
$$

Evidently, the current $\left(\gamma^{\mu}\right)$ terms are divergent, whereas the magnetic $\left(\sigma^{\mu \nu}\right)$ terms are finite. Within the SM, the term containing $z_{\mathrm{L}}^{q}$ has been calculated previously using unitary gauge in Refs. [2, 3], and our expression above agrees with the results found therein.

In writing down $\mathcal{M}_{d \rightarrow d^{\prime} Z^{*}}^{q}$, we have dropped finite terms (without $\Delta$ ) that do not depend on the internal quark mass $m_{q}$, or equivalently $x_{q}=m_{q}^{2} / m_{W}^{2}$. This is because such terms will be removed by the Glashow-Iliopoulos-Maiani (GIM) mechanism in the SM, after summing over $q=u, c, t$ and imposing the unitarity relation $V_{u d^{\prime}}^{*} V_{u d}+V_{c d^{\prime}}^{*} V_{c d}+V_{t d^{\prime}}^{*} V_{t d}=0$, and by a GIM-like mechanism beyond the SM. For the same reason, we also drop $m_{q}$-independent finite terms in the next two amplitudes for $d \rightarrow d^{\prime} \mathcal{V}^{*}$, as well as in the box-diagram contributions below.

For $\mathcal{V}=\gamma$, after combining the three contributions (a,b,c), we find that the $k$-independent terms cancel completely, but that terms of first and second orders in $k$ do not, which we keep, namely

$$
\mathcal{M}_{d \rightarrow d^{\prime} \gamma^{*}}^{q}=\frac{e \varepsilon_{\mu}^{*}}{16 \pi^{2} m_{W}^{2}} \bar{d}^{\prime}\left[\left(k^{2} \gamma^{\mu}-\not k k^{\mu}\right)\left(f_{\mathrm{L}}^{q} P_{\mathrm{L}}+f_{\mathrm{R}}^{q} P_{\mathrm{R}}\right)+i \sigma^{\mu \nu} k_{\nu}\left(F_{\mathrm{L}}^{q} P_{\mathrm{L}}+F_{\mathrm{R}}^{q} P_{\mathrm{R}}\right)\right] d
$$

where

$$
\begin{aligned}
f_{\mathrm{L}}^{q}=\frac{g_{\mathrm{L}}^{d} \bar{g}_{\mathrm{L}}^{d^{\prime}}}{4}\left[\left(x_{q}-\frac{16}{3}\right) \Delta+\frac{153 x_{q}-383 x_{q}^{2}+245 x_{q}^{3}-27 x_{q}^{4}}{18\left(1-x_{q}\right)^{3}}\right. & \\
& \left.+\frac{-16+64 x_{q}-36 x_{q}^{2}-93 x_{q}^{3}+84 x_{q}^{4}-9 x_{q}^{5}}{9\left(1-x_{q}\right)^{4}} \ln x_{q}\right] \\
f_{\mathrm{R}}^{q}= & \frac{g_{\mathrm{R}}^{d} \bar{g}_{\mathrm{R}}^{d^{\prime}}}{g_{\mathrm{L}}^{d} \bar{g}_{\mathrm{L}}^{d^{\prime}}} f_{\mathrm{L}}^{q}, \\
F_{\mathrm{L}}^{q}= & 2\left(g_{\mathrm{L}}^{d} \bar{g}_{\mathrm{L}}^{d^{\prime}} m_{d^{\prime}}+g_{\mathrm{R}}^{d} \bar{g}_{\mathrm{R}}^{d^{\prime}} m_{d}\right) F_{0}^{\mathrm{SM}}\left(x_{q}\right)+2 g_{\mathrm{L}}^{d} \bar{g}_{\mathrm{R}}^{d^{\prime}} m_{q} F_{0}\left(x_{q}\right) \\
F_{\mathrm{R}}^{q}= & 2\left(g_{\mathrm{L}}^{d} \bar{g}_{\mathrm{L}}^{d^{\prime}} m_{d}+g_{\mathrm{R}}^{d} \bar{g}_{\mathrm{R}}^{d^{\prime}} m_{d^{\prime}}\right) F_{0}^{\mathrm{SM}}\left(x_{q}\right)+2 \bar{g}_{\mathrm{L}}^{d^{\prime}} g_{\mathrm{R}}^{d} m_{q} F_{0}\left(x_{q}\right), \\
& F_{0}^{\mathrm{SM}}(x)=\frac{-7 x+5 x^{2}+8 x^{3}}{24(1-x)^{3}}-\frac{2 x^{2}-3 x^{3}}{4(1-x)^{4}} \ln x \\
& F_{0}(x)=\frac{-20+31 x-5 x^{2}}{12(1-x)^{2}}-\frac{2 x-3 x^{2}}{2(1-x)^{3}} \ln x
\end{aligned}
$$

This amplitude also contains terms which are divergent, but its magnetic part is finite, as in the $\mathcal{V}=Z$ case. The $f_{\mathrm{L}}^{q}$ term has been evaluated in unitary gauge before [2, 3, 4], and our result 
confirms those given in Refs. [2, 3], ${ }^{1}$ but differs from that in Ref. [4]. The magnetic, $F_{\mathrm{L}, \mathrm{R}}^{q}$, terms have also been evaluated in unitary gauge previously in Refs. [4, 5], and the resulting expressions are consistent with ours, at linear order in $g_{\mathrm{R}}$.

For $\mathcal{V}$ being a gluon, $g_{a}$, the diagram in Fig. 1(b) is absent, and we get

$$
\mathcal{M}_{d \rightarrow d^{\prime} g_{a}^{*}}^{q}=\frac{g_{\mathrm{s}} \varepsilon_{\mu}^{*}}{16 \pi^{2} m_{W}^{2}} \bar{d}^{\prime}\left[\left(k^{2} \gamma^{\mu}-\not k k^{\mu}\right)\left(g_{\mathrm{L}}^{q} P_{\mathrm{L}}+g_{\mathrm{R}}^{q} P_{\mathrm{R}}\right)+i \sigma^{\mu \nu} k_{\nu}\left(G_{\mathrm{L}}^{q} P_{\mathrm{L}}+G_{\mathrm{R}}^{q} P_{\mathrm{R}}\right)\right] t_{a} d,
$$

where

$$
\begin{gathered}
g_{\mathrm{L}}^{q}=\frac{g_{\mathrm{L}}^{d} \bar{g}_{\mathrm{L}}^{d^{\prime}}}{g_{\mathrm{R}}^{d} \bar{g}_{\mathrm{R}}^{d^{\prime}}} g_{\mathrm{R}}^{q}=\frac{g_{\mathrm{L}}^{d} \bar{g}_{\mathrm{L}}^{d^{\prime}}}{4}\left[\frac{18 x_{q}-11 x_{q}^{2}-x_{q}^{3}}{3\left(1-x_{q}\right)^{3}}-\frac{8-32 x_{q}+18 x_{q}^{2}}{3\left(1-x_{q}\right)^{4}} \ln x_{q}\right], \\
G_{\mathrm{L}}^{q}=2\left(g_{\mathrm{L}}^{d} \bar{g}_{\mathrm{L}}^{d^{\prime}} m_{d^{\prime}}+g_{\mathrm{R}}^{d} \bar{g}_{\mathrm{R}}^{d^{\prime}} m_{d}\right) G_{0}^{\mathrm{SM}}\left(x_{q}\right)+2 g_{\mathrm{L}}^{d} \bar{g}_{\mathrm{R}}^{d^{\prime}} m_{q} G_{0}\left(x_{q}\right), \\
G_{\mathrm{R}}^{q}=2\left(g_{\mathrm{L}}^{d} \bar{g}_{\mathrm{L}}^{d^{\prime}} m_{d}+g_{\mathrm{R}}^{d} \bar{g}_{\mathrm{R}}^{d^{\prime}} m_{d^{\prime}}\right) G_{0}^{\mathrm{SM}}\left(x_{q}\right)+2 \bar{g}_{\mathrm{L}}^{d^{\prime}} g_{\mathrm{R}}^{d} m_{q} G_{0}\left(x_{q}\right),
\end{gathered}
$$

with

$$
G_{0}^{\mathrm{SM}}(x)=\frac{-2 x-5 x^{2}+x^{3}}{8(1-x)^{3}}-\frac{3 x^{2} \ln x}{4(1-x)^{4}}, \quad G_{0}(x)=\frac{-4-x-x^{2}}{4(1-x)^{2}}-\frac{3 x \ln x}{2(1-x)^{3}} .
$$

Unlike in the $\mathcal{V}=Z$ and $\gamma$ cases, this amplitude has no divergence. Our expressions for the terms containing $g_{\mathrm{L}}^{q}$ and $G_{0}^{\mathrm{SM}}$ in the SM limit agree with the corresponding unitary-gauge results of Ref. [6], except for the relative sign between them. The magnetic part containing $G_{0}$ has been previously calculated in unitary gauge in Ref. [7], but the result therein misses a factor of $\frac{1}{2}$.

Before proceeding to evaluate the box diagrams, we note that the divergent terms in $\mathcal{M}_{d \rightarrow d^{\prime} Z^{*}}^{q}$ and $\mathcal{M}_{d \rightarrow d^{\prime} \gamma^{*}}^{q}$ above depend on $x_{q}$. This implies that in the SM limit,

$$
g_{\mathrm{L}}^{d}=\frac{g}{\sqrt{2}} V_{q d}, \quad \bar{g}_{\mathrm{L}}^{d^{\prime}}=\frac{g}{\sqrt{2}} V_{q d^{\prime}}^{*}, \quad g_{\mathrm{R}}^{d}=g_{\mathrm{R}}^{d^{\prime}}=0
$$

these divergences cannot be eliminated by the GIM mechanism. We have checked that such divergences would still be present in the absence of the second diagram in Fig. 1(b), which has a greater degree of divergence than the others. In contrast, the corresponding terms calculated in $R_{\xi}$ gauges are finite [8, 9, 10]. The $k^{2} \gamma^{\mu}-\not k k^{\mu}$ parts of the $\mathcal{V}=Z, \gamma$ amplitudes are, therefore, gauge dependent. For physical processes, such as $d \bar{d}^{\prime} \rightarrow \nu \bar{\nu}$ and $d \bar{d}^{\prime} \rightarrow \ell^{+} \ell^{-}$, we will show later that the $x_{q}$-dependent divergences contributed by the $d \rightarrow d^{\prime} \mathcal{V}^{*}$ diagrams in unitary gauge are canceled exactly by the $x_{q}$-dependent divergences in the box-diagram contributions. The magnetic $\left(\sigma^{\mu \nu}\right)$ terms in the $d \rightarrow d^{\prime} \mathcal{V}^{*}$ amplitude are, on the other hand, gauge independent. In particular, our expressions for the $F_{\mathrm{L}, \mathrm{R}}^{q}$ and $G_{\mathrm{L}, \mathrm{R}}^{q}$ terms (at first order in $g_{\mathrm{R}}$ ) agree with those derived using $R_{\xi}$ gauges [9, 10, 11]

We also note that the divergent terms in $\mathcal{M}_{d \rightarrow d^{\prime} Z^{*}}^{q}$ will also be present in the amplitude for the physical decay $Z \rightarrow \bar{d} d^{\prime}$. In that case, $k^{2}$-dependent terms need to be kept and, as a consequence,

${ }^{1}$ A factor of $x_{t}$ seems to be missing from the $T^{\text {div }}$ term in the Eq. (25) of Ref. [3] 
there are additional divergent contributions. The divergent part of the $d \rightarrow d^{\prime} Z^{*}$ amplitude in the $k^{2} \neq 0$ case is then

$$
\begin{aligned}
\mathcal{M}_{d \rightarrow d^{\prime} Z^{*}}^{q,(\text { div })}=\frac{g \Delta}{16 \pi^{2}} \bar{d}^{\prime} \nexists^{*}\{ & \frac{g_{\mathrm{L}}^{d} \bar{g}_{\mathrm{L}}^{d^{\prime}}}{4 c_{\mathrm{w}}}\left[\left(\frac{c_{\mathrm{w}}^{2} k^{2}}{m_{W}^{2}}-1\right) x_{q}-\frac{\left(16 m_{W}^{2}+k^{2}\right) c_{\mathrm{w}}^{2} k^{2}}{3 m_{W}^{4}}\right] P_{\mathrm{L}} \\
& \left.+\frac{g_{\mathrm{R}}^{d} \bar{g}_{\mathrm{R}}^{d^{\prime}}}{4 c_{\mathrm{w}}}\left[\left(\frac{c_{\mathrm{w}}^{2} k^{2}}{m_{W}^{2}}+7\right) x_{q}-\frac{\left(16 m_{W}^{2}+k^{2}\right) c_{\mathrm{w}}^{2} k^{2}}{3 m_{W}^{4}}\right] P_{\mathrm{R}}\right\} d,
\end{aligned}
$$

where the $k^{2}$-dependent terms have been supplied by the second diagram in Fig. 1. Accordingly, setting $k^{2}=m_{Z}^{2}=m_{W}^{2} / c_{\mathrm{w}}^{2}$, one finds for $Z \rightarrow \bar{d} d^{\prime}$

$$
\mathcal{M}_{Z \rightarrow \bar{d} d^{\prime}}^{q, \operatorname{div}}=\frac{g \Delta}{192 \pi^{2} c_{\mathrm{w}}^{3}} \bar{d}^{\prime} \not \subset\left[-g_{\mathrm{L}}^{d} \bar{g}_{\mathrm{L}}^{d^{\prime}}\left(16 c_{\mathrm{w}}^{2}+1\right) P_{\mathrm{L}}+g_{\mathrm{R}}^{d} \bar{g}_{\mathrm{R}}^{d^{\prime}}\left(24 c_{\mathrm{w}}^{2} x_{q}-16 c_{\mathrm{w}}^{2}-1\right) P_{\mathrm{R}}\right] d
$$

Clearly, in the SM limit the $m_{q}$-dependent part of the divergence in the $Z \rightarrow \bar{d} d^{\prime}$ amplitude vanishes, but the remaining $x_{q}$-independent part is removed only after applying the GIM mechanism. This is in accord with what is found in the literature [12].

In evaluating the box diagrams, we assume that the $W$ has SM couplings to leptons and set the masses and momenta of the external fermions to zero. Thus, from Fig. 2(a) we obtain

$$
\begin{aligned}
\mathcal{M}_{d \bar{d}^{\prime} \rightarrow \nu \bar{\nu}}^{q, \text { box }}= & \frac{g^{2} g_{\mathrm{L}}^{d} \bar{g}_{\mathrm{L}}^{d^{\prime}}}{128 \pi^{2} m_{W}^{2}}\left[\left(x_{q}+x_{\ell}-6\right) \Delta+\mathcal{B}_{2}\left(x_{q}, x_{\ell}\right)\right] \bar{d}^{\prime} \gamma^{\mu} P_{\mathrm{L}} d \bar{\nu} \gamma_{\mu} P_{\mathrm{L}} \nu \\
& +\frac{g^{2} g_{\mathrm{R}}^{d} \bar{g}_{\mathrm{R}}^{d^{\prime}}}{128 \pi^{2} m_{W}^{2}}\left[\left(x_{q}+x_{\ell}-6\right) \Delta+\mathcal{B}_{1}\left(x_{q}, x_{\ell}\right)\right] \bar{d}^{\prime} \gamma^{\mu} P_{\mathrm{R}} d \bar{\nu} \gamma_{\mu} P_{\mathrm{L}} \nu \\
\mathcal{M}_{d \bar{d}^{\prime} \rightarrow \ell^{+} \ell^{-}}^{q, \text { box }} & =\frac{g^{2} g_{\mathrm{L}}^{d} \bar{g}_{\mathrm{L}}^{d^{\prime}}}{128 \pi^{2} m_{W}^{2}}\left[\left(6-x_{q}\right) \Delta-\mathcal{B}_{1}\left(x_{q}, 0\right)\right] \bar{d}^{\prime} \gamma^{\mu} P_{\mathrm{L}} d \bar{\ell} \gamma_{\mu} P_{\mathrm{L}} \ell \\
& +\frac{g^{2} g_{\mathrm{R}}^{d} \bar{g}_{\mathrm{R}}^{d^{\prime}}}{128 \pi^{2} m_{W}^{2}}\left[\left(6-x_{q}\right) \Delta-\mathcal{B}_{2}\left(x_{q}, 0\right)\right] \bar{d}^{\prime} \gamma^{\mu} P_{\mathrm{R}} d \bar{\ell} \gamma_{\mu} P_{\mathrm{L}} \ell
\end{aligned}
$$

where

$$
\begin{gathered}
\mathcal{B}_{1}(x, y)=\frac{3}{2}(x+y)+\frac{3(x+y-x y)}{(1-x)(1-y)}+\frac{\left(4 x^{2}-8 x^{3}+x^{4}\right) \ln x}{(y-x)(1-x)^{2}}+\frac{\left(4 y^{2}-8 y^{3}+y^{4}\right) \ln y}{(x-y)(1-y)^{2}} \\
\mathcal{B}_{2}(x, y)=\frac{3}{2}(x+y)-\frac{9(x+y-x y)}{(1-x)(1-y)}+\frac{(4-x)^{2} x^{2} \ln x}{(y-x)(1-x)^{2}}+\frac{(4-y)^{2} y^{2} \ln y}{(x-y)(1-y)^{2}}
\end{gathered}
$$

and $\ell$ in Eq. (20) refers to the charged lepton in the loop. Since the divergences in these box contributions depend on $m_{q}$, they cannot be removed by the GIM mechanism in the SM limit, Eq. (17). This is different from the corresponding contributions computed in the $R_{\xi}$ gauge, which are finite [9] 
Combining the $d \bar{d}^{\prime} \rightarrow Z^{*} \rightarrow \nu \bar{\nu}$ amplitude derived from Eq. (44) and the box-diagram amplitude in Eq. (20), we then find

$$
\begin{aligned}
\mathcal{M}_{d \bar{d}^{\prime} \rightarrow \nu \bar{\nu}}^{q} & =\frac{g^{2} g_{\mathrm{L}}^{d} \bar{g}_{\mathrm{L}}^{d^{\prime}}}{32 \pi^{2} m_{W}^{2}}\left[\frac{\left(x_{\ell}-6\right) \Delta}{4}+4 X_{0}\left(x_{q}\right)+\frac{\mathcal{B}_{2}\left(x_{q}, x_{\ell}\right)-\mathcal{B}_{2}\left(x_{q}, 0\right)}{4}\right] \bar{d}^{\prime} \gamma^{\mu} P_{\mathrm{L}} d \bar{\nu} \gamma_{\mu} P_{\mathrm{L}} \nu \\
& +\frac{g^{2} g_{\mathrm{R}}^{d} \bar{g}_{\mathrm{R}}^{d^{\prime}}}{32 \pi^{2} m_{W}^{2}}\left[\left(2 x_{q}+\frac{x_{\ell}-6}{4}\right) \Delta+\tilde{X}\left(x_{q}\right)+\frac{\mathcal{B}_{1}\left(x_{q}, x_{\ell}\right)-\mathcal{B}_{1}\left(x_{q}, 0\right)}{4}\right] \bar{d}^{\prime} \gamma^{\mu} P_{\mathrm{R}} d \bar{\nu} \gamma_{\mu} P_{\mathrm{L}} \nu
\end{aligned}
$$

where

$$
X_{0}(x)=\frac{x(x+2)}{8(x-1)}+\frac{3 x(x-2)}{8(x-1)^{2}} \ln x, \quad \tilde{X}(x)=2 x-\frac{5 x-2 x^{2}}{1-x} \ln x-4 X_{0}(x) .
$$

In writing down this amplitude, we have neglected the contribution of the $Z$-magnetic dipole terms, with $Z_{\mathrm{L}, \mathrm{R}}^{q}$, in Eq. (4), which are small compared to the $z_{\mathrm{L}, \mathrm{R}}^{q}$ terms due to the $m_{W}$ suppression. We can see that in the SM limit, Eq. (17), the divergent part of $\mathcal{M}_{d \bar{d}^{\prime} \rightarrow \nu \bar{\nu}}^{q}$ no longer depends on $m_{q}$ because of cancelation between the $m_{q}$-dependent divergent terms in the $Z$-penguin and box contributions. The remaining divergence will then disappear when the GIM mechanism operates.

For $d \bar{d}^{\prime} \rightarrow \ell^{+} \ell^{-}$, the amplitude receives not only the $Z$-mediated and box contributions, but also the photon-mediated one derived from Eq. (8) . As a result

$$
\begin{aligned}
\mathcal{M}_{d \bar{d}^{\prime} \rightarrow \ell^{+} \ell^{-}}^{q}= & \frac{g^{2} g_{\mathrm{L}}^{d} \bar{g}_{\mathrm{L}}^{d^{\prime}}}{32 \pi^{2} m_{W}^{2}}\left(\frac{3 \Delta}{2}-4 Y_{0}\left(x_{q}\right)\right) \bar{d}^{\prime} \gamma^{\mu} P_{\mathrm{L}} d \bar{\ell} \gamma_{\mu} P_{\mathrm{L}} \ell \\
& +\frac{g^{2} g_{\mathrm{L}}^{d} \bar{g}_{\mathrm{L}}^{d^{\prime}} s_{\mathrm{w}}^{2}}{32 \pi^{2} m_{W}^{2}}\left(-\frac{8 \Delta}{3}+8 Z_{0}\left(x_{q}\right)\right) \bar{d}^{\prime} \gamma^{\mu} P_{\mathrm{L}} d \bar{\ell} \gamma_{\mu} \ell \\
& +\frac{g^{2} g_{\mathrm{R}}^{d} \bar{g}_{\mathrm{R}}^{d^{\prime}}}{32 \pi^{2} m_{W}^{2}}\left[\left(\frac{3}{2}-2 x_{q}\right) \Delta+\tilde{Y}\left(x_{q}\right)\right] \bar{d}^{\prime} \gamma^{\mu} P_{\mathrm{R}} d \bar{\ell} \gamma_{\mu} P_{\mathrm{L}} \ell \\
& +\frac{g^{2} g_{\mathrm{R}}^{d} \bar{g}_{\mathrm{R}}^{d^{\prime}} s_{\mathrm{w}}^{2}}{32 \pi^{2} m_{W}^{2}}\left[\left(4 x_{q}-\frac{8}{3}\right) \Delta+\tilde{Z}\left(x_{q}\right)\right] \bar{d}^{\prime} \gamma^{\mu} P_{\mathrm{R}} d \bar{\ell} \gamma_{\mu} \ell \\
& +\frac{i g^{2} s_{\mathrm{w}}^{2}}{16 \pi^{2} m_{W}^{2} k^{2}} \bar{d}^{\prime} \sigma^{\mu \nu} k_{\nu}\left(F_{\mathrm{L}}^{q} P_{\mathrm{L}}+F_{\mathrm{R}}^{q} P_{\mathrm{R}}\right) d \bar{\ell} \gamma_{\mu} \ell
\end{aligned}
$$

where

$$
\begin{gathered}
Y_{0}(x)=\frac{x(x-4)}{8(x-1)}+\frac{3 x^{2}}{8(x-1)^{2}} \ln x \\
Z_{0}(x)=\frac{18 x^{4}-163 x^{3}+259 x^{2}-108 x}{144(x-1)^{3}}+\frac{24 x^{4}-6 x^{3}-63 x^{2}+50 x-8}{72(x-1)^{2}} \ln x, \\
\tilde{Y}(x)=-2 x+\frac{5 x-2 x^{2}}{1-x} \ln x+4 Y_{0}(x), \quad \tilde{Z}(x)=2 x-4 x \ln x+8 Z_{0}(x),
\end{gathered}
$$

and we have again neglected the contribution of the $Z$-magnetic dipole terms in Eq. (4). In the SM limit, the divergent terms in the total amplitude are also independent of $m_{q}$ due to cancelation 
among the $m_{q}$-dependent divergent parts of the penguin and box contributions. This, along with the similar cancelation in the $d \bar{d}^{\prime} \rightarrow \nu \bar{\nu}$ case, explicitly confirms the result of unitary-gauge analysis in Ref. [13].

In terms of the couplings $\kappa_{q d}^{\mathrm{L}, \mathrm{R}}$ parameterizing the anomalous interactions of quarks and the $W$ boson considered in Ref. [1], we have

$$
g_{\mathrm{L}}^{d}=\frac{g}{\sqrt{2}} V_{q d}\left(1+\kappa_{q d}^{\mathrm{L}}\right), \quad g_{\mathrm{R}}^{d}=\frac{g}{\sqrt{2}} V_{q d} \kappa_{q d}^{\mathrm{R}},
$$

where the $\kappa$ 's are assumed to be small compared to unity. It is then straightforward to arrive at the effective Hamiltonians for $d \bar{d}^{\prime} \rightarrow \nu \bar{\nu}$ and $d \bar{d}^{\prime} \rightarrow \ell^{+} \ell^{-}$within the SM, as well as those induced by the anomalous couplings, given in Ref. [1].

For $d \bar{d}^{\prime} \rightarrow \bar{d} d^{\prime}$, we derive the amplitude from the two box-diagrams in Fig. 2 with quarks $d$ and $d^{\prime} \neq d$ in the external legs and quarks $q$ and $q^{\prime}$ in the loops. In this case we adopt the parametrization in Eq. (28). With the contribution of the anomalous couplings included to second order in $\kappa$, it follows that

$$
\begin{aligned}
\mathcal{M}_{d \bar{d}^{\prime} \rightarrow \bar{d} d^{\prime}}^{q}= & \frac{g^{4} \lambda_{q}^{\mathrm{L}} \lambda_{q^{\prime}}^{\mathrm{L}}}{256 \pi^{2} m_{W}^{2}}\left[\left(6-x_{q}-x_{q^{\prime}}\right) \Delta-\mathcal{B}_{1}\left(x_{q}, x_{q^{\prime}}\right)\right] \bar{d}_{1}^{\prime} \gamma^{\alpha} P_{\mathrm{L}} d_{1} \bar{d}_{2}^{\prime} \gamma_{\alpha} P_{\mathrm{L}} d_{2} \\
& +\frac{g^{4} \lambda_{q} \lambda_{q^{\prime}}}{256 \pi^{2} m_{W}^{2}}\left[\left(6-x_{q}-x_{q^{\prime}}\right) \Delta-\mathcal{B}_{2}\left(x_{q}, x_{q^{\prime}}\right)\right] \\
& \times\left(\kappa_{q d^{\prime}}^{\mathrm{R} *} \kappa_{q d}^{\mathrm{R}} \bar{d}_{1}^{\prime} \gamma^{\alpha} P_{\mathrm{L}} d_{1} \bar{d}_{2}^{\prime} \gamma_{\alpha} P_{\mathrm{R}} d_{2}+\kappa_{q^{\prime} d^{\prime}}^{\mathrm{R} *} \kappa_{q^{\prime} d}^{\mathrm{R}} \bar{d}_{1}^{\prime} \gamma^{\alpha} P_{\mathrm{R}} d_{1} \bar{d}_{2}^{\prime} \gamma_{\alpha} P_{\mathrm{L}} d_{2}\right) \\
& -\frac{g^{4} \lambda_{q} \lambda_{q^{\prime}} m_{q} m_{q^{\prime}}}{64 \pi^{2} m_{W}^{4}}\left(\Delta+\mathcal{B}_{3}\left(x_{q}, x_{q^{\prime}}\right)\right)\left(\kappa_{q d^{2}}^{\mathrm{R}} \kappa_{q^{\prime} d}^{\mathrm{R}} \bar{d}_{1}^{\prime} P_{\mathrm{R}} d_{1} \bar{d}_{2}^{\prime} P_{\mathrm{R}} d_{2}+\kappa_{q d^{\prime}}^{\mathrm{R} *} \kappa_{q^{\prime} d^{\prime}}^{\mathrm{R} *} \bar{d}_{1}^{\prime} P_{\mathrm{L}} d_{1} \bar{d}_{2}^{\prime} P_{\mathrm{L}} d_{2}\right) \\
& +\left(d_{1}^{\prime} \leftrightarrow d_{2}^{\prime}\right)
\end{aligned}
$$

where we have distinguished the two $d^{(\prime)}$ 's,

$$
\begin{gathered}
\lambda_{q}^{\mathrm{L}}=\lambda_{q}\left(1+\kappa_{q d^{\prime}}^{\mathrm{L} *}\right)\left(1+\kappa_{q d}^{\mathrm{L}}\right), \quad \lambda_{q}^{\mathrm{R}}=\lambda_{q} \kappa_{q d^{\prime}}^{\mathrm{R} *} \kappa_{q d}^{\mathrm{R}}, \\
\mathcal{B}_{3}(x, y)=\frac{x y-x-y-2}{(1-x)(1-y)}+\frac{\left(4 x-2 x^{2}+x^{3}\right) \ln x}{(y-x)(1-x)^{2}}+\frac{\left(4 y-2 y^{2}+y^{3}\right) \ln y}{(x-y)(1-y)^{2}}
\end{gathered}
$$

and we have neglected finite terms quadratic in $\kappa^{\mathrm{R}}$ containing $\bar{d}^{\prime} \sigma^{\mu \nu} P_{\mathrm{L}, \mathrm{R}} d \bar{d}^{\prime} \sigma_{\mu \nu} P_{\mathrm{L}, \mathrm{R}} d$ whose contributions to the neutral-meson mixing would vanish in the vacuum saturation approximation. It is also straightforward to obtain the corresponding effective Hamiltonians given in Ref. [1]. This $d \bar{d}^{\prime} \rightarrow \bar{d} d^{\prime}$ amplitude in unitary gauge has been previously calculated in Ref. [14], but our result for the $\kappa^{\mathrm{R}}$ terms disagrees with theirs.

Finally, we remark that some of the physical amplitudes we have found above generated by the anomalous couplings contain divergent parts. As discussed in Ref. [1], this is due to the fact that the effective theory with anomalous couplings in Eq. (11) is not renormalizable, and the divergences are understood in the context of effective field theories as contributions to the coefficients of higherdimension operators, which are not included in our analysis. 
In conclusion, we have provided the detailed derivation of loop formulas summarized in our recent work evaluating several two- and four-fermion FCNC transitions induced at one-loop level by the anomalous charm- $W$ couplings. In treating the loop diagrams, we have worked in unitary gauge and used dimensional regularization to handle the divergences arising in some of the loops. We have compared our results with the corresponding ones obtained in the literature using unitary and $R_{\xi}$ gauges, where available. In particular, we have discussed subtleties in the cancelation of divergences from individual contributions to some of the physical amplitudes and derived expressions consistent with those obtained in $R_{\xi}$ gauges. Finally, we have provided, perhaps for the first time, the unitary-gauge expressions for the separate diagrams contributing to the $d \rightarrow d^{\prime} \mathcal{V}^{*}$ amplitude. These particular results can be applied to other models involving fermions and gauge bosons with interactions similar in form to those considered here. For example, the contribution of the $Z^{\prime}$ boson to the $b \rightarrow s \gamma$ transition can be easily found from the magnetic terms in our general expression for the $d \rightarrow d^{\prime} \mathcal{V}^{*}$ amplitude, with appropriate couplings.

\section{Acknowledgments}

The work of X.G.H. and J.T. was supported in part by NSC and NCTS. The work of G.V. was supported in part by DOE under contract number DE-FG02-01ER41155.

\section{APPENDIX A: AMPLITUDES FOR $d \rightarrow d^{\prime} \mathcal{V}^{*}$}

The Lagrangian describing a neutral gauge-boson $\mathcal{V}$ coupling to a fermion-antifermion pair, $f \bar{f}$, or a $W$-boson pair can be expressed as

$$
\mathcal{L}_{\mathcal{V}}=-\bar{f} \gamma^{\mu}\left(L_{f}^{\mathcal{\nu}} P_{\mathrm{L}}+R_{f}^{\mathcal{V}} P_{\mathrm{R}}\right) f \mathcal{V}_{\mu}+i g_{W}^{\mathcal{V}}\left[\mathcal{V}^{\mu}\left(W_{\mu \nu}^{+} W^{-\nu}-W^{+\nu} W_{\mu \nu}^{-}\right)-\mathcal{V}^{\mu \nu} W_{\mu}^{+} W_{\nu}^{-}\right],
$$

where $L_{f}^{\mathcal{V}}, R_{f}^{\mathcal{V}}$, and $g_{W}^{\mathcal{V}}$ contain the coupling constants, and $X_{\mu \nu}=\partial_{\mu} X_{\nu}-\partial_{\nu} X_{\mu}$. This and $\mathcal{L}_{U D W}$ in Eq. (1) provide the relevant vertices for the $d \rightarrow d^{\prime} \mathcal{V}^{*}$ transition at one-loop level, leading to the diagrams in Fig. 1, where the external quarks $d$ and $d^{\prime}$ are on-shell and $\mathcal{V}$ is off-shell. The resulting amplitudes for the contributions (a,b,c), with $q$ being the quark in the loops and $\mathcal{V}$ having a four-momentum $k$, are, respectively,

$$
\begin{aligned}
\mathcal{M}_{d \rightarrow d^{\prime} \mathcal{V}^{*}}^{(\mathrm{a})}=\frac{g_{\mathrm{L}}^{d} \bar{g}_{\mathrm{L}}^{d^{\prime}}}{16 \pi^{2}} \bar{d}^{\prime} & {\left[\left(\frac{R_{q}^{\mathcal{V}}-4 L_{q}^{\mathcal{V}}}{2} x_{q} \Delta+L_{q}^{\mathcal{V}} V_{1}\left(x_{q}\right)+R_{q}^{\mathcal{V}} V_{2}\left(x_{q}\right)\right) \nexists^{*} P_{\mathrm{L}}\right.} \\
& +\left(L_{q}^{\mathcal{V}} V_{3}\left(x_{q}\right)+R_{q}^{\mathcal{V}} V_{4}\left(x_{q}\right)\right) \frac{k^{2} \nexists^{*}-\not k \varepsilon^{*} \cdot k}{m_{W}^{2}} P_{\mathrm{L}} \\
& +\left(L_{q}^{\mathcal{V}} V_{5}\left(x_{q}\right)+R_{q}^{\mathcal{V}} V_{6}\left(x_{q}\right)\right)\left(m_{d^{\prime}} P_{\mathrm{L}}+m_{d} P_{\mathrm{R}}\right) \frac{i \sigma^{\mu \nu} \varepsilon_{\mu}^{*} k_{\nu}}{m_{W}^{2}} \\
& \left.+\frac{\bar{g}_{\mathrm{R}}^{d^{\prime}}}{\bar{g}_{\mathrm{L}}^{d^{\prime}}}\left(L_{q}^{\mathcal{V}}+R_{q}^{\mathcal{V}}\right) V_{7}\left(x_{q}\right) m_{q} P_{\mathrm{L}} \frac{i \sigma^{\mu \nu} \varepsilon_{\mu}^{*} k_{\nu}}{m_{W}^{2}}\right] d \\
+\left(g_{\mathrm{L}} \leftrightarrow\right. & \left.g_{\mathrm{R}}, L_{q}^{\mathcal{V}} \leftrightarrow R_{q}^{\mathcal{V}}, P_{\mathrm{L}} \leftrightarrow P_{\mathrm{R}}\right),
\end{aligned}
$$




$$
\begin{aligned}
& \mathcal{M}_{d \rightarrow d^{\prime} \mathcal{V}^{*}}^{(\mathrm{b}) q}=\frac{g_{W}^{\mathcal{V}}}{16 \pi^{2}} \bar{d}^{\prime}\{\left(\frac{3 x_{q} \Delta}{2}-V_{1}\left(x_{q}\right)-V_{2}\left(x_{q}\right)\right) \nexists^{*}\left(g_{\mathrm{L}}^{d} \bar{g}_{\mathrm{L}}^{d^{\prime}} P_{\mathrm{L}}+g_{\mathrm{R}}^{d} \bar{g}_{\mathrm{R}}^{d^{\prime}} P_{\mathrm{R}}\right) \\
&+\left(\frac{3 x_{q}-16}{12} \Delta+V_{8}\left(x_{q}\right)\right) \frac{k^{2} \nexists^{*}-\not k \varepsilon^{*} \cdot k}{m_{W}^{2}}\left(g_{\mathrm{L}}^{d} \bar{g}_{\mathrm{L}}^{d^{\prime}} P_{\mathrm{L}}+g_{\mathrm{R}}^{d} \bar{g}_{\mathrm{R}}^{d^{\prime}} P_{\mathrm{R}}\right) \\
&+V_{9}\left(x_{q}\right)\left[\left(g_{\mathrm{L}}^{d} \bar{g}_{\mathrm{L}}^{d^{\prime}} m_{d^{\prime}}+g_{\mathrm{R}}^{d} \bar{g}_{\mathrm{R}}^{d^{\prime}} m_{d}\right) P_{\mathrm{L}}+\left(g_{\mathrm{L}}^{d} \bar{g}_{\mathrm{L}}^{d^{\prime}} m_{d}+g_{\mathrm{R}}^{d} \bar{g}_{\mathrm{R}}^{d^{\prime}} m_{d^{\prime}}\right) P_{\mathrm{R}}\right] \frac{i \sigma^{\mu \nu} \varepsilon_{\mu}^{*} k_{\nu}}{m_{W}^{2}} \\
&\left.+V_{10}\left(x_{q}\right) m_{q}\left(g_{\mathrm{L}}^{d} \bar{g}_{\mathrm{R}}^{d^{\prime}} P_{\mathrm{L}}+g_{\mathrm{L}}^{d^{\prime}} \bar{g}_{\mathrm{R}}^{d} P_{\mathrm{R}}\right) \frac{i \sigma^{\mu \nu} \varepsilon_{\mu}^{*} k_{\nu}}{m_{W}^{2}}\right\} d \\
& \mathcal{M}_{d \rightarrow d^{\prime} \mathcal{V}^{*}}^{(\mathrm{c}) q}=\frac{1}{16 \pi^{2}}\left(\frac{3 x_{q} \Delta}{2}-V_{1}\left(x_{q}\right)-V_{2}\left(x_{q}\right)\right) \bar{d}^{\prime} \nexists^{*}\left(g_{\mathrm{L}}^{d} \bar{g}_{\mathrm{L}}^{d^{\prime}} L_{d}^{\mathcal{V}} P_{\mathrm{L}}+g_{\mathrm{R}}^{d} \bar{g}_{\mathrm{R}}^{d^{\prime}} R_{d}^{\mathcal{V}} P_{\mathrm{R}}\right) d
\end{aligned}
$$

where $\Delta$ is given in Eq. (3),$\varepsilon$ and $k$ are the polarization and four-momentum of $\mathcal{V}$, respectively,

$$
\begin{aligned}
& \bar{g}_{\mathrm{L}}^{d^{\prime}}=\left(g_{\mathrm{L}}^{d^{\prime}}\right)^{*}, \quad \bar{g}_{\mathrm{R}}^{d^{\prime}}=\left(g_{\mathrm{R}}^{d^{\prime}}\right)^{*}, \quad x_{f}=\frac{m_{f}^{2}}{m_{W}^{2}}, \quad \sigma^{\mu \nu}=\frac{i}{2}\left[\gamma^{\mu}, \gamma^{\nu}\right], \\
& V_{1}(x)=-\frac{3}{2}-x+2 x \ln x, \quad V_{2}(x)=\frac{-7 x+x^{2}}{4(1-x)}-\frac{4 x-2 x^{2}+x^{3}}{2(1-x)^{2}} \ln x \\
& V_{3}(x)=-\frac{2}{9}+\frac{10 x-5 x^{2}-11 x^{3}}{36(1-x)^{3}}-\frac{(2-3 x)^{2}}{6(1-x)^{4}} \ln x \\
& V_{4}(x)=\frac{1}{3}+\frac{11 x-7 x^{2}+2 x^{3}}{9(1-x)^{3}}+\frac{2 x \ln x}{3(1-x)^{4}}, \\
& V_{5}(x)=\frac{2}{3}+\frac{5 x-22 x^{2}-5 x^{3}}{12(1-x)^{3}}+\frac{x-3 x^{2}}{2(1-x)^{4}} \ln x, \quad V_{6}(x)=\frac{1}{4}-\frac{3}{4} V_{4}(x) \\
& V_{7}(x)=-1-\frac{9 x-3 x^{2}}{4(1-x)^{2}}-\frac{3 x \ln x}{2(1-x)^{3}}, \\
& V_{8}(x)=\frac{25}{18}+\frac{27 x-113 x^{2}+83 x^{3}-9 x^{4}}{24(1-x)^{3}}-\frac{31 x^{3}-28 x^{4}+3 x^{5}}{12(1-x)^{4}} \ln x, \\
& V_{9}(x)=\frac{5}{6}-\frac{x-5 x^{2}-2 x^{3}}{4(1-x)^{3}}+\frac{3 x^{3} \ln x}{2(1-x)^{4}}, \quad V_{10}(x)=6 V_{4}(x)+V_{5}(x)+3 V_{7}(x)-2 V_{9}(x) .
\end{aligned}
$$

In obtaining these results, we have assumed that $k$ and $m_{d, d^{\prime}}$ are small compared to $m_{W}$, kept terms to second order in $k$, and taken at the end of the calculation the usual limit of vanishing $m_{d, d^{\prime}}$ for the nonleading terms [8, 9]. It is worth noting that the $x_{q}$-dependent part of the divergent term in each of these amplitudes arises from the $p^{\mu} p^{\nu}$ terms of the $W$ propagators.

The separate expressions above for $\mathcal{M}_{d \rightarrow d^{\prime} \mathcal{V}^{*}}^{(\mathrm{a}, \mathrm{b}) q}$ in unitary gauge may have been written down for the first time in this work. They are general and follow from any effective interactions of the form given in Eqs. (11) and (A1), provided that the masses of the external fermions and the momentum 
of the external gauge boson are much less than the gauge-boson mass in the loops. In the case of the standard model, the couplings of $\mathcal{V}=Z, \gamma, g_{a}$ in $\mathcal{L}_{\mathcal{V}}$ are parametrized by

$$
\begin{gathered}
L_{f}^{Z}=\frac{g}{c_{\mathrm{w}}}\left(I_{3 f}-s_{\mathrm{w}}^{2} Q_{f}\right), \quad R_{f}^{Z}=-\frac{g}{c_{\mathrm{w}}} s_{\mathrm{w}}^{2} Q_{f}, \\
L_{f}^{\gamma}=R_{f}^{\gamma}=e Q_{f}, \quad L_{f}^{g_{a}}=R_{f}^{g_{a}}=g_{\mathrm{s}} t_{a}, \\
g_{W}^{Z}=c_{\mathrm{w}} g, \quad g_{W}^{\gamma}=e=g s_{\mathrm{w}}, \quad g_{W}^{g_{a}}=0, \\
c_{\mathrm{w}}=\cos \theta_{\mathrm{W}}, \quad s_{\mathrm{w}}=\sin \theta_{\mathrm{W}},
\end{gathered}
$$

where $I_{3 f}$ and $Q_{f}$ are as usual the third component of weak isospin of $f$ and its electric charge, respectively, and $t_{a}$ are the color-SU(3) generators satisfying $\operatorname{Tr}\left(t_{a} t_{b}\right)=\frac{1}{2} \delta_{a b}$.

[1] X.G. He, J. Tandean, and G. Valencia, Phys. Rev. D 80, 035021 (2009) [arXiv:0904.2301 [hep-ph]].

[2] A.J. Buras, A. Poschenrieder, S. Uhlig, and W.A. Bardeen, JHEP 0611, 062 (2006) arXiv:hep-ph/0607189; M. Blanke et al., JHEP 0701, 066 (2007) arXiv:hep-ph/0610298.

[3] L.S. Wu and Z.J. Xiao, Commun. Theor. Phys. 48, 502 (2007) arXiv:hep-ph/0612326].

[4] S.P. Chia and G. Rajagopal, Phys. Lett. B 156, 405 (1985); S.P. Chia, Phys. Lett. B 240, 465 (1990).

[5] K. Fujikawa and A. Yamada, Phys. Rev. D 49, 5890 (1994).

[6] S.P. Chia, Phys. Lett. B 130, 315 (1983).

[7] A. Abd El-Hady and G. Valencia, Phys. Lett. B 414, 173 (1997) arXiv:hep-ph/9704300.

[8] E. Ma and A. Pramudita, Phys. Rev. D 22, 214 (1980).

[9] T. Inami and C.S. Lim, Prog. Theor. Phys. 65, 297 (1981) [Erratum-ibid. 65, 1772 (1981)].

[10] N.G. Deshpande and G. Eilam, Phys. Rev. D 26, 2463 (1982); N.G. Deshpande and M. Nazerimonfared, Nucl. Phys. B 213, 390 (1983); J.M. Soares and A. Barroso, Phys. Rev. D 39, 1973 (1989).

[11] P.L. Cho and M. Misiak, Phys. Rev. D 49, 5894 (1994) arXiv:hep-ph/9310332.

[12] A. Axelrod, Nucl. Phys. B 209, 349 (1982); M. Clements, C. Footman, A.S. Kronfeld, S. Narasimhan, and D. Photiadis, Phys. Rev. D 27, 570 (1983); V. Ganapathi, T.J. Weiler, E. Laermann, I. Schmitt, and P.M. Zerwas, Phys. Rev. D 27, 579 (1983); G. Mann and T. Riemann, Annalen Phys. 40, 334 (1984).

[13] V.I. Kuksa, Phys. Atom. Nucl. 66, 1737 (2003) [Yad. Fiz. 66, 1785 (2003)].

[14] J.P. Lee and K.Y. Lee, Phys. Rev. D 78, 056004 (2008) arXiv:0806.1389 [hep-ph]]. 